\section{Persistência dos sintomas de dengue em uma população de Uberaba, Minas Gerais, Brasil}

\author{
Persistence of dengue symptoms in patients \\ in Uberaba, Minas Gerais State, Brazil
}

\begin{abstract}
The clinical picture of dengue is characterized by a maximum duration of 14 days despite frequent complaints of longer symptoms. This study evaluated the occurrence of persistent symptoms (> 14 days) and its impact on daily life. A hundred eighteen patients were interviewed, and the main symptoms at diagnosis were mialgia (98.3\%), fever (97.5\%) and weakness (95.8\%). The presence of at least a persistent symptom was related by 77 (65.2\%) individuals of wich 10 (8.5\%) described it as intense and lasting for 30 days or more. The most persistent symptoms mentioned were weakness (58 cases), hiporexia (49) and sleepiness (40), occurring mostly in women, with odds ratio: 5.4 (IC95\%: 2.3-12.3). A significant association between the persistence of the symptoms and the history of extra expenses ( $p=0,02)$ was found, as well as a delay to return to normal activities $(p<$ 0.001). Thus, it was verified that dengue presented a relevant impact on every day life, even after 14 days, a fact wich was associated with the presence of persistent symptoms of the illness.
\end{abstract}

Dengue; Signs and Symptoms; Sickness Impact Profile
Luciana de Almeida Silva Teixeira 1 Juliana Salviano Mendonça Lopes 1 André Guilherme da Costa Martins 1 Fernando Augusto Batista Campos 1 Sybelle de Souza Castro Miranzi 1 Gabriel Antônio Nogueira Nascentes 1

\section{Introdução}

Dengue é uma arbovirose responsável por importante impacto na saúde pública, principalmente nos países em desenvolvimento. Foi estimado o gasto médio por indivíduo em 514 dólares para pacientes ambulatoriais e de 1.394 dólares para os hospitalizados 1 .

Na maioria dos casos a infecção é autolimitada e dura cerca de 14 dias 2, no entanto, estudos demonstraram sintomas a longo prazo, como fadiga, miosite persistente e rabdomiólise $3,4,5$. Assim, o dengue pode cursar com manifestações clínicas prolongadas, embora ainda não sejam bem conhecidas, bem como seu impacto na vida economicamente ativa dos indivíduos infectados.

O número expressivo de casos de dengue ocorridos em Uberaba, Minas Gerais, no ano de 2006, aliado aos freqüentes relatos de sintomas da doença acima do prazo considerado habitual, serviram de motivação para realização deste estudo, cujos objetivos foram: caracterizar as manifestações clínicas do dengue; verificar a ocorrência de manifestações prolongadas (> 14 dias) e avaliar o impacto causado pela doença na vida útil dos pacientes. 


\section{Casuística e métodos}

Trata-se de estudo observacional do tipo inquérito transversal, com base em informação retrospectiva.

A identificação dos casos de dengue notificados em Uberaba foi obtida no Departamento de Vigilância em Saúde da Secretaria Municipal de Saúde. Baseando-se nesse banco de dados foi feito o cálculo amostral, estimando-se a confirmação de $60 \%$ dos casos (com base na proporção dos anos anteriores), com intervalo de $90 \%$ de confiança e valor de $\mathrm{p}=0,01$.

$$
\begin{gathered}
\mathrm{n}=\frac{\mathrm{Z}^{2} \cdot \mathrm{p} \cdot(1-\mathrm{p})=(1,64)^{2} \cdot 0,011 \cdot(1-0,011)}{\mathrm{D}^{2}}= \\
\frac{293+20 \%(59)=352 \text { casos }}{(0,01)^{2}}
\end{gathered}
$$

Os 352 endereços foram selecionados aleatoriamente dentro do banco, por meio do programa Epi Info (Centers for Disease Control and Prevention, Atlanta, Estados Unidos). Todos foram visitados e destes, 45 (12,7\%) não foram encontrados, 108 (30,6\%) indivíduos estavam ausentes; $61(17,3 \%)$ não residiam no local e 21 (6\%) não foram entrevistados por outros motivos. Portanto, 118 foram convidados e assinaram o termo de consentimento livre e esclarecido. As entrevistas ocorreram entre outubro de 2007 e abril de 2008 , e foram feitas com base em questionário semiestruturado contendo variáveis sociodemográficas, sanitárias e clínico-epidemiológicas:

a) Sociodemográficas: gênero, escolaridade, idade, raça, ocupação, renda mensal, religião, tipo de habitação, estado conjugal, trabalho atual, dias de trabalho e aula perdidos devido ao dengue e gastos adicionais referidos pelo paciente;

b) Sanitárias: viagem antecedente à doença, local da viagem, terreno baldio no entorno, saneamento, presença de plantas em casa, visita do agente de saúde e reconhecimento do Aedes aegypti;

c) Clínico-epidemiológicas: foram divididas em sintomas do início da doença e os que persistiram por mais de 14 dias. Assim, foram interrogados objetivamente os sintomas de febre, cefaléia, dor retro-orbitária, mialgia, exantema, prostração, artralgia, náuseas, vômitos, diarréia, adenopatia, fraqueza, dor abdominal, hiporexia, perda de memória e sonolência. Além disso, foram perguntados a data do diagnóstico de dengue, evidência laboratorial, história familiar da doença, antecedente pessoal e vacinação contra febre amarela.

Os dados foram analisados nos programas SPSS 16.0 (SPSS Inc., Chicago, Estados Unidos) ou Statistica 7.0 (Statsoft Inc.; http://www.stat soft.com), utilizando-se o qui-quadrado e o cálculo do odds ratio (OR) com intervalo de $95 \%$ de confiança (IC95\%).

O estudo foi aprovado pelo Comitê de Ética em Pesquisa da Universidade Federal do Triângulo Mineiro (protocolo 951).

\section{Resultados}

Dos 118 entrevistados, 102 (86,4\%) apresentaram o diagnóstico de dengue confirmado por sorologia. Houve predominância da faixa etária entre 20 e 60 anos (72,9\%), gênero feminino $(66,1 \%)$ estado civil casado $(47,4 \%)$, escolaridade igual ou inferior ao Ensino Fundamental completo $(66,1 \%)$ e religião católica $(58,5 \%)$. A maioria dos indivíduos residia em casa $(97,4 \%)$, com renda familiar variável e saneamento básico presente em todos os imóveis. A presença de terreno baldio no entorno, bem como de plantas em casa foi referida pela maioria dos entrevistados $(68,6 \%$ e $75,4 \%$, respectivamente). Quanto às medidas educativas, $109(92,4 \%)$ receberam visitas de agentes de saúde da Prefeitura, com o intuito de promover medidas educativas, e $88(74,6 \%)$ referiram reconhecer o vetor (Tabela 1 ).

O maior número de casos da doença foi registrado nos meses de fevereiro e março, e 90 (76,3\%) indivíduos confirmaram a presença de outros casos na família.

Foi verificado que 60 (50,8\%) foram vacinados contra a febre amarela há menos de 10 anos; $35(29,7 \%)$ há mais de 10 anos e 23 (19,5\%) nunca foram imunizados ou não souberam responder.

Em relação ao quadro clínico inicial de dengue, os sintomas mais prevalentes foram mialgia $(98,3 \%)$, febre $(97,5 \%)$, fraqueza $(95,8 \%)$, prostração $(94,1 \%)$ e cefaléia $(92,4 \%)$ (Tabela 2$)$.

Considerando os sintomas com duração superior a 14 dias, $77(65,2 \%)$ entrevistados referiram a presença de pelo menos um deles, sendo que $10(8,5 \%)$ relataram sintomas intensos por 30 dias ou mais. Os sintomas persistentes mais mencionados foram fraqueza, hiporexia e sonolência (Tabela 3).

Quanto à condução dos casos, 16 (13,6\%) pessoas foram internadas, todas por menos de 14 dias. O uso de medicação foi referido por 112 (94,9\%) entrevistados, sendo que, destes, 99 usaram paracetamol e 33 dipirona. A maioria $(79,5 \%)$ referiu uso de medicamento por menos de 14 dias.

A dificuldade de retorno às atividades habituais depois de 15 dias do início da doença, associada à necessidade de afastamento, foi referida por 68 (57,6\%) entrevistados. Decorridos 60 dias, 
Tabela 1

Distribuição dos indivíduos segundo as variáveis relativas à caracterização sociodemográfica.

\section{Dados sociodemográficos}

Indivíduos

$\%$

Idade (anos)
$<20$
$21-40$
$41-60$
$>61$

Gênero

Feminino

Masculino

40

Estado civil

Solteiro

Casado

Amasiado

Viúvo

Outro

11,0

Escolaridade

Analfabeto ou ensino fundamental incompleto

Ensino Fundamental completo ou ensino médio incompleto

Ensino Médio completo

Ensino Superior

Religião

Católico

Espírita

Evangélico

Sem religião

33,0

39

47,4

56

7,6

9

5,9

7

7

5,9

enda familiar (salário mínimo)

$<1$

1

$1-2$

2-3

$>3$

Não informou

46,6

$23 \quad 19,5$

$31 \quad 26,3$

9

Tipo de habitação

Casa

Apartamento

Saneamento básico

Sim

97,4

115

2,6

Não

Terreno baldio no entorno

Sim

0

0,0

Não

Presença de plantas no domicílio

Sim

Não

$89 \quad 75,5$

22

18,6

Não informaram

7

5,9

(continua) 
Tabela 1 (continuação)

\begin{tabular}{lcc}
\hline Dados sociodemográficos & n & Indivíduos \\
& & $\%$ \\
Reconhece o Aedes aegypti & 88 & 74,6 \\
Sim & 23 & 19,5 \\
Não & 7 & 5,9 \\
Não responderam & & 109 \\
Recebe visita do agente de saúde & 5 & 92,4 \\
Sim & 4 & 3,2 \\
Não & 4 & 3,4 \\
Não respondeu
\end{tabular}

Tabela 2

Freqüência de sinais e sintomas ocorridos no momento do diagnóstico de dengue.

\begin{tabular}{lcc}
\hline Sintomas & \multicolumn{2}{c}{ Número de casos } \\
& $\mathbf{n}$ & $\%$ \\
\hline Mialgia & 116 & 98,3 \\
Febre & 115 & 97,5 \\
Fraqueza & 113 & 95,8 \\
Prostração & 111 & 94,1 \\
Cefaléia & 109 & 92,4 \\
Artralgia & 102 & 86,8 \\
Dor retro-orbitária & 87 & 73,7 \\
Náuseas & 86 & 72,9 \\
Exantema & 64 & 54,2 \\
Vômitos & 63 & 53,4 \\
Diarréia & 44 & 37,3 \\
Adenopatia & 22 & 18,6 \\
Outros & 48 & 40,7 \\
\hline
\end{tabular}

$14(11,9 \%)$ persistiram com dificuldades leve ou moderada, e, após seis meses, 6 (5,1\%) ainda referiam dificuldade leve de retorno às atividades cotidianas. Gastos adicionais foram relatados por $91(77,1 \%)$ pacientes, sendo $98,9 \%$ relacionados com medicação.

Não houve associação entre a presença de sintomas persistentes de dengue e a vacinação contra febre amarela $(p=0,539)$.

Os indivíduos que relataram sintomas persistentes de febre e perda de memória apresentavam média de idade superior (53,7 e 52,2 anos, respectivamente) aos que não relataram estes sintomas $(40,4$ e 40,3, respectivamente) $(\mathrm{p}=$ 0,044 e $p=0,043$ ). Além disso, o gênero feminino foi associado aos sintomas persistentes ( $\mathrm{p}<$ 0,001) com OR de 5,38 (IC95\%: 2,35-12,34) em relação ao masculino.
A maioria dos pacientes com sintomas persistentes $(84,2 \%)$ referiu gastos adicionais, com associação significativa entre as duas variáveis ( $\mathrm{p}=0,023$ ). Assim, os indivíduos que apresentaram sintomas persistentes tiveram chance 2,76 vezes maior (IC95\%: 1,13-6,75) de terem gastos adicionais, quando comparados aos indivíduos sem tais sintomas.

A presença de sintomas persistentes também foi associada à dificuldade de retorno às atividades habituais $(\mathrm{p}<0,001)$. Os indivíduos que apresentaram sintomas persistentes mostraram chance 18,52 vezes maior (IC95\%: 6,93-49,45) de apresentar alguma dificuldade de retorno. Ademais, os pacientes que apresentaram dificuldade tiveram chance 5,81 vezes superior (IC95\%: 2,21 $15,25)$ de terem gastos com a doença em relação aos indivíduos que retornaram às atividades sem complicações $(\mathrm{p}<0,001)$.

\section{Discussão}

No presente estudo, verificou-se predominância de indivíduos que residiam em casa, com plantas e terreno baldio no entorno e maior prevalência da doença nos períodos chuvosos. Esses dados confirmam a situação epidemiológica favorável já descrita para os surtos de dengue ${ }^{2}$. Ressaltamos os relatos das visitas de agentes de saúde com função educativa, e a maioria dos entrevistados conhecer o vetor da doença. Ainda assim, tais medidas de controle pareceram não impedir o surgimento de casos nesses domicílios. Essa percepção tem sido confirmada em outros estudos, ao se evidenciar que as medidas educativas nem sempre implicam a mudança de atitude da população ${ }^{6}$.

O quadro clínico inicial do dengue também seguiu o padrão habitual de apresentação da doença. Cabe ressaltar que os sintomas não são específicos e podem ser confundidos com outras 
Sintomas persistentes de dengue classificados conforme sua intensidade e duração, por número de pacientes acometidos.

\begin{tabular}{|c|c|c|c|c|c|c|c|}
\hline \multirow[t]{2}{*}{ Sintomas persistentes } & \multirow{2}{*}{$\begin{array}{l}\text { Número de } \\
\text { indivíduos }\end{array}$} & \multicolumn{3}{|c|}{ Intensidade } & \multicolumn{3}{|c|}{ Duração } \\
\hline & & Leve & Moderada & Intensa & $15-30$ dias & $31-45$ dias & $>45$ dias \\
\hline Fraqueza & 58 & 32 & 16 & 10 & 41 & 3 & 14 \\
\hline Hiporexia & 49 & 27 & 14 & 8 & 41 & 2 & 6 \\
\hline Sonolência & 40 & 20 & 11 & 9 & 31 & 2 & 7 \\
\hline Mialgia & 32 & 22 & 7 & 3 & 20 & 2 & 10 \\
\hline Cefaléia & 26 & 16 & 3 & 7 & 22 & - & 4 \\
\hline Dor retro-orbitária & 17 & 8 & 2 & 7 & 14 & - & 3 \\
\hline Dor abdominal & 12 & 9 & 1 & 2 & 9 & 2 & 1 \\
\hline Perda de memória & 9 & 5 & 4 & - & 7 & - & 2 \\
\hline Febre & 7 & 5 & - & 2 & 6 & - & 1 \\
\hline Náuseas ou vômitos & 5 & 4 & 1 & - & 2 & 1 & 2 \\
\hline Diarréia & 1 & 1 & - & - & 1 & - & - \\
\hline
\end{tabular}

doenças febris agudas. Na amostragem deste estudo, a maioria $(86,4 \%)$ apresentou confirmação sorológica. Ademais, a apresentação clínica e a presença de sintomas persistentes não foram significativamente diferentes entre os casos confirmados e os não confirmados. Por esses motivos, optou-se pela inclusão do número total de entrevistados.

A presença de pelo menos um sintoma persistente de dengue foi referida por mais de $60 \%$ dos entrevistados. Chama atenção o fato dos pacientes atribuírem os sintomas ao quadro de dengue e que não os apresentavam anteriormente. Os mais freqüentes foram sintomas inespecíficos, mas que persistiram de maneira intensa por 30 dias ou mais, em um número significativo de pacientes.

Foi verificada a associação entre presença de sintomas persistentes e gênero feminino, bem como à idade acima de 50 anos. Esses achados concordam com outros autores que associaram a idade e o gênero feminino à ocorrência de fadiga pós-infecção pelo dengue 3 .

A vacinação prévia contra a febre amarela não se associou aos sintomas persistentes de dengue, sugerindo que não há imunização cruzada entre as duas doenças.
A associação entre a presença de sintomas persistentes e os gastos adicionais com a doença, aliada à dificuldade de retorno às atividades habituais, confirmam a interferência do dengue na vida economicamente ativa do indivíduo acometido. Esse impacto seria subestimado se levássemos em conta apenas o tempo considerado habitual de duração (até 14 dias).

Como limitações deste estudo, ressalta-se o reduzido número de indivíduos incluídos na pesquisa e o tempo transcorrido entre a doença e a entrevista. Todos os 352 endereços foram visitados, momento no qual se verificaram erros de registro ou que os pacientes haviam mudado do local. Além disso, como o estudo se baseou em entrevista retrospectiva, o tempo entre a doença e a entrevista pode interferir na memória dos pacientes e, por conseguinte, na precisão dos dados fornecidos.

Finalmente, os resultados permitem concluir que o dengue apresentou interferência na vida útil dos indivíduos, associada à presença de sintomas persistentes, afastamento das atividades regulares e gastos adicionais. Este assunto merece investimento de estudos posteriores que permitam avaliar o impacto do dengue de forma prospectiva e, assim, direcionar futuras ações de saúde coletiva destinadas a minimizar também os danos individuais. 


\section{Resumo}

O quadro clínico clássico de dengue caracteriza-se por duração máxima de 14 dias, embora freqüentemente pacientes refiram prolongamento dos sintomas. Este estudo avaliou a ocorrência de sinais e sintomas persistentes (>14 dias) e seu impacto no cotidiano do indivíduo doente. Foram entrevistados 118 pacientes, cujos principais sintomas ao diagnóstico foram mialgia (98,3\%), febre (97,5\%) e fraqueza (95,8\%). A presença de pelo menos um sintoma persistente foi referida por $77(65,2 \%)$ entrevistados, sendo que $10(8,5 \%)$ relataram sua permanência de maneira intensa e por 30 dias ou mais. Os sintomas persistentes mais mencionados foram fraqueza (58 casos), hiporexia (49) $e$ sonolência (40), ocorrendo mais no gênero feminino, com odds ratio: 5,4 (IC95\%: 2,3-12,3). Houve associação significativa entre a persistência dos sintomas e o relato de gastos adicionais $(p=0,02)$, e com o retorno às atividades habituais $(p<0,001)$. Assim, foi verificado que o dengue apresentou impacto na vida dos indivíduos, mesmo depois de 14 dias, sendo tal fato associado à presença de sintomas persistentes da doença.

Dengue; Sinais e Sintomas; Perfil de Impacto da Doença

\section{Colaboradores}

L. A. S. Teixeira participou de todas as etapas, desde a elaboração do projeto até a redação final do artigo. J. S. M. Lopes participou na elaboração do projeto, coleta de dados, análise e redação do trabalho. A. G. C. Martins e F. A. B. Campos participaram na coleta de dados, análise dos resultados e redação do trabalho. S. S. C. Miranzi participou na concepção, viabilização e redação do trabalho. G. A. N. Nascentes participou na análise estatística, interpretação dos resultados e na redação científica do artigo.

\section{Agradecimentos}

À Universidade Federal do Triângulo Mineiro, por viabilizar transporte para realização das entrevistas, e à Secretaria Municipal de Saúde de Uberaba pela disponibilização dos dados de notificação.

\section{Referências}

1. Suaya JA, Shepard DS, Siqueira JB, Martelli CT, Lum LC, Tan LH, et al. Cost of dengue cases in eight countries in the Americas and Asia: a prospective study. Am J Trop Med Hyg 2009; 80:846-55.

2. Halstead SB. Dengue. Lancet 2007; 370:1644-52.

3. Seet RCS, Quek AML, Lim ECH. Post-infectious fatigue syndrome in dengue infection. J Clin Virol 2007; 38:1-6.

4. Finsterer J, Kongchan K. Severe, persisting, steroid-responsive Dengue myositis. J Clin Virol 2006; 35:426-8.
5. Lim M, Goh HK. Rhabdomyolysis following dengue virus infection. Singapore Med J 2005; 46:645-6.

6. Donalisio MR, Alves MJCP, Visockas A. Inquérito sobre conhecimentos e atitudes da população sobre a transmissão do dengue - região de Campinas, São Paulo, Brasil - 1998. Rev Soc Bras Med Trop 2001; 34:197-201.

Recebido em 02/Mai/2009

Versão final reapresentada em 12/Jan/2010 Aprovado em 02/Fev/2010 